\title{
Coverage and invariability in fuzzy systems.
}

\author{
Miguel Lloret-Climent, Josué-Antonio Nescolarde-Selva and Sergio Pérez-Gonzaga \\ Department of Applied Mathematics, University of Alicante, Alicante, Spain
}

\begin{abstract}
This paper presents a new complex system systemic. Here, we are working in a fuzzy environment, so we have to adapt all the previous concepts and results that were obtained in a non-fuzzy environment, for this fuzzy case. The direct and indirect influences between variables will provide the basis for obtaining fuzzy and/or non-fuzzy relationships, so that the concepts of coverage and invariability between sets of variables will appear naturally. These two concepts and their interconnections will be analyzed from the viewpoint of algebraic properties of inclusion, union and intersection (fuzzy and non-fuzzy), and also for the loop concept, which, as we shall see, will be of special importance.
\end{abstract}

Keywords: Complex system, coverage, fuzzy relations; fuzzy coverage, fuzzy invariability.

\section{Introduction}

In many complex problems arising in the fields of engineering, medical science, economics, social sciences, etc. involving uncertainties, exact methods have recently been found to be inadequate. Many theories have been proposed for dealing with uncertainties efficiently, such as Zadeh's fuzzy sets theory (1965), Atanassov's intuitionistic fuzzy sets theory (1986), and Pawlak's rough fuzzy sets theory (1982). In 1999, Molodtsov (1999) introduced the concept of the soft set which is a new approach for modeling uncertainties. In 2013, Gunduz (Aras) and Bayramov introduced important properties of fuzzy soft topological spaces.

When studying complex problems, developing plans, managing organizations and in other kinds of human endeavor, it is often essential to synthesize hierarchies. Based on fuzzy sets theory, Tazaki and Amagasa (1979) propose a method for structuring hierarchy for the various problems in managing organizations, i.e. Fuzzy Structural Modeling (FSM). This method describes a formal procedure for constructing the graphic presentation of the hierarchical arrangement, given the necessary information concerning the relation of each element to every other element. Hierarchy is fundamental to many fields of science, engineering and human situations. Raghuvanshi and Kumar (1999) present a generalized fuzzy structural modeling (GFSM) allowing the inclusions of cycles of any order.

Since people often decide on their behavior without a definite sense of their motives, fuzzy theory should be an effective tool for assisting people in making decisions. Fuzzy theory should be an effective tool to develop a system to provide psychological support for human decision-making. Yamashita (1997) proposes a support system which is composed of a combination of the fuzzy reasoning model and fuzzy structural modeling (FSM).

Multicriteria fuzzy decision-making problems (Chen and Tan 1994; Hong and Choi 2000) have been proposed based on the vague set theory. Liu and Wang (2007) proposed multicriteria decision-making methods based on Atanassov's (1986) intuitionistic fuzzy sets, Ye (2010) proposed a fuzzy decision-making method based on the weighted correlation coefficient in an intuitionistic fuzzy environment by means of entropy weights. Recently, Ye (2012) established measures based on the Hamming and Euclidean distances between intuitionistic trapezoidal fuzzy numbers to solve the multicriteria group decision-making problem.

The evaluation process of a location selection problem involves a multiplicity of complex considerations and poses a multi-criteria decision-making (MCDM) situation. Moreover, 
some evaluation criteria faced ambiguous and uncertain nature. Ding and Chou (2013) develop a fuzzy (MCDM) model to evaluate the best selection of transshipment ports for container carriers.

When constructing a probability distribution from incomplete and imprecise data, the effects of the quantity and the quality of the data are of serious concern in practical applications. Kikuchi and Kronprasert (2012) present an approach to building a complete probability distribution from an incomplete probability distribution, when some of the given probabilities are only approximately known.

Several approaches to the idea of fuzzy graph have been introduced in the literature on fuzzy systems. Graph theory has many applications for problems in systems analysis, operations research, economics and transportation, but some aspects of a graph-theoretic problem may be uncertain in many situations. Blue et al. (2002) present a taxonomy of fuzzy graphs, providing a catalog of the various types of "fuzziness" of possible graphs.

Fuzzy logic has developed into an extensive subject. Zadeh (1999) addresses the terminology, and stresses that fuzzy graphs are a generalization of the calculi of crisp graphs. Other formulations of fuzzy graph problems have appeared in the literature; Kóczy (1992) also gives a taxonomy of fuzzy graphs.

In this paper, we present a new systemic approach to complex systems, extending the results obtained in previous works (Esteve and Lloret 2006a,b), by using a fuzzy set theory. The direct and indirect influences between variables will provide the basis for obtaining fuzzy and/or non-fuzzy relationships, so that the concepts of coverage and invariability between sets of variables will appear naturally, these concepts and their interconnections will be analyzed from the viewpoint of algebraic properties of inclusion, union and intersection, as well as for the loop concept, which as we shall see, will be especially important. In our work, we use some important concepts of the fuzzy system, including membership function, inclusion, intersection, union, support and fuzzy relation.

\section{Fuzzy influences}

When any two elements are related, we know that one of them exercises, directly, a certain influence on the other. Taking this into account, we obtain the following definitions and results, bearing in mind the fact that the relations will always be fuzzy from now on since we can always consider a classic relation as a particular case of a fuzzy one.

Definition 1. In a complex system $S=(M, R)$, if two elements $a, b \in M$ verify that $\exists r \in R$, $\mu_{r}(a, b)=\mathrm{p}>0$ for any fuzzy relation, we say that $a$ directly influences $b$ with grade $p$ through the fuzzy relation $r$.

From now on, we need to bear in mind the fact that the chains of relations that will exist between any two elements of $\mathrm{M}$ may contain classic or fuzzy relations of the types mentioned here in indiscriminate fashion, as well as containing elements that could belong to fuzzy sets or not.

Clearly, all the degrees of influence we are going to consider will strictly be greater than zero. If not, we would have superfluous influences, so we eliminate them and simplify the chain of relations. 
With these premises, only considering the degree of influence and not how it is produced, we will now formalize the concept of indirect influence, first presenting the concept of the chain of relations, something that will be of great use:

Definition 2. Let $S=(M, R)$ be a complex system. We shall say that a set of variables $\left\{x_{1}, \ldots\right.$, $\left.x_{n}\right\}$ form a chain of relations if there is a set of relations (fuzzy or not) $\left\{r_{1}, \ldots, r_{n-1}\right\} \in R$ such that $\left\{x_{1} r_{1} x_{2}, x_{2} r_{2} x_{3}, \ldots, x_{n-1} r_{n-1} x_{n}\right\}$.

From the above we obtain:

Definition 3. In a complex system $S=(M, R)$, we say that the element $a \in M$ indirectly influences the element $b \in M$ with grade $p$, if there is a chain, starting with $a$ and ending with $b$, that contains relations of $R$ and elements of $M$, and verifying that the minimum degree of indirect influence obtained with this chain is equal to $p$.

Of all the chains of relations, special importance is attributed, due to the role it plays in the systemic analysis of any complex system, to what we know as the loop:

Definition 4. In a complex system $S=(M, R)$ we say that a set $\left\{x_{i}\right\}_{i=1}^{n} \subseteq M$ is a grade $p$ loop for the relations (fuzzy or not) $r_{1}, r_{2}, \ldots, r_{n} \in R$ if we verify that:

$$
\mu_{r 1}\left(x_{1}, x_{2}\right)=\mathrm{p}_{1}>0, \mu_{r 2}\left(x_{2}, x_{3}\right)=\mathrm{p}_{2}>0, \ldots \ldots, \mu_{r n}\left(x_{n}, x_{1}\right)=\mathrm{p}_{\mathrm{n}}>0 \text { y } \mathrm{p}=\min \left(\mathrm{p}_{1}, \mathrm{p}_{2}, \ldots, \mathrm{p}_{\mathrm{n}}\right) .
$$

In the trivial case, a set $\{x\} \subseteq M$ is a grade $p$ single loop for the fuzzy relation $r \in R$ if $\mu_{r}(x, x)=\mathrm{p}>0$. Meaning that $x$ has a direct influence on itself with grade $\mathrm{p}$.

From the definition of the loop we obtain another of greater importance:

Definition 5. In a complex system $S=(M, R)$ we say that the structure of the system is hierarchical if no subset of $M$ is a loop.

When we focus on a specific variable, its behavior is immediately defined formally as follows:

Definition 6. In a complex system $S=(M, R)$ we define the relations structure of each variable $x \in M$ as $f_{M}(x)=\left\{y \in M / \exists r \in \mathrm{R}, \mu_{r}(x, y)>0\right\}$.

Definition 7. In a complex system $S=(M, R)$ we define the relations structure of the variable $x \in M$ associated with the fuzzy relation $r \in R$ as $f_{M}(x, r)=\left\{y \in M / \mu_{r}(x, y)>0\right\}$.

If we were interested in a set of variables, the previous definitions would be as follows:

Definition 8. In a complex system $S=(M, R)$ we define the relations structure of any set $X \subseteq M$ by $f_{M}(X)=\bigcup_{x \in X} f_{M}(x)$.

Definition 9. In a complex system $S=(M, R)$ we define the relations structure of any set $X \subseteq M$ and associated with the fuzzy relation $r \in R$ by $f_{M}(X, r)=\bigcup_{x \in X} f_{M}(x, r)$.

From the above we can deduce the following result: 
Proposition 1. In a complex system $S=(M, R)$, for each $X \subseteq M$ we verify that $f_{M}(X)=\bigcup_{r \in R} f_{M}(X, r)$.

Proof. $\bigcup_{r \in R} f_{M}(X, r)=\bigcup_{r \in R}\left(\bigcup_{x \in X} f_{M}(x, r)\right)=\bigcup_{x \in X}\left(\bigcup_{r \in R} f_{M}(x, r)\right)=\bigcup_{x \in X} f_{M}(x)=f_{M}(X)$.

\section{Coverage}

One of the first properties to appear when studying systemic approach of complex systems is coverage (Esteve and Lloret, 2006a,b). This property is fundamental when studying the direct relations existing between all the subsets of $M$. It also provides criteria for determining the existence of loops. We now offer an example to illustrate adaptation to a fuzzy environment and an initial result of interest:

Definition 10. Let $S=(M, R)$ be a complex system and let $A, B \subseteq M$. We say that $A$ covers $B$ if $f_{M}(A)=B$. In other words, if each element of $B$ is directly influenced by any element of $A$.

For the case where the sets $A$ and $B$ are fuzzy, the above definition becomes $f_{M}(\operatorname{supp}(A))=\operatorname{supp}(B)$, as for any variable $a \in A$ whose membership function is 0 , we obtain $\mu_{r}(a, b) \leq \min \left(\mu_{A}(a), \mu_{B}(b)\right)=\min \left(0, \mu_{B}(b)\right)=0$, so that $\mu_{r}(a, b)=0$, and $a$ will not be effectively related to any other variable.

However, if set $A$ is fuzzy and $B$ is non-fuzzy, the definition of coverage becomes:

$f_{M}(\operatorname{supp}(A))=B$.

We now present a practical example to clarify the above concept:

Example 1. Let the complex system $S=(M, R)$ defined on the set of individuals $M=\left\{x_{1}, x_{2}, x_{3}, x_{4}, x_{5}, x_{6}, x_{7}\right\}$.

Let the fuzzy set $B=$ "'Individual that may suffer from ischemic cardiopathy" with $\operatorname{supp}(B)=$ $=\left\{x_{1}, x_{4}, x_{7}\right\}$, with the degrees of membership being $\mu_{B}\left(x_{1}\right)=0,8 ; \mu_{B}\left(x_{4}\right)=0,7 ; \mu_{B}\left(x_{7}\right)=0,9$.

In addition, let the following risk factors associated with illness:

$A_{1}=$ "Suffers from high blood pressure"

$A_{2}=$ "Has a high BMI (body mass index)"

$A_{3}=$ "Heavy smoker"

$A_{4}=$ "Sedentary lifestyle"

$A_{5}=$ "Suffers from dyslipemia"

We construct a fuzzy set $A$ that integrates all these risk factors, weighting the degree of membership of each of the sets according to the impact of the risk factor.

We thus obtain the following degrees of membership:

$\operatorname{Supp}(A)=\left\{x_{1}, x_{2}, x_{3}, x_{4}, x_{5}, x_{6}, x_{7}\right\}$ where $\mu_{A}\left(x_{1}\right)=0.8 ; \mu_{A}\left(x_{2}\right)=0.7 ; \quad \mu_{A}\left(x_{3}\right)=0.9 ; \quad \mu_{A}\left(x_{4}\right)=0.5$;

$\mu_{A}\left(x_{5}\right)=0.1 ; \mu_{A}\left(x_{6}\right)=0.4 ; \mu_{A}\left(x_{7}\right)=0.9$.

We construct the fuzzy relation $r$ as follows: $r=\left\{(a, b) / a, b \in M, \mu_{r}(a, b)=\min \left(\mu_{A}(a), \mu_{B}(b)\right\}\right.$.

In this case, $f_{M}(\operatorname{supp}(A))=\operatorname{supp}(B)$, meaning that the fuzzy set $A$ covers fuzzy set $B$, meaning that the fuzzy set made up of the individuals associated with risk factors for ischemic cardiopathy covers the set of individuals that really suffer from it.

Proposition 2. Let $S=(M, R)$ be a complex system and let the fuzzy sets be $A, B, C \subseteq M$. If $A$ covers $B$ and $B$ covers $C$, then each element of $\operatorname{supp}(C)$ is indirectly influenced by an element of $\operatorname{supp}(A)$. 
Proof. Let $c \in \operatorname{supp}(C)$. As $B$ covers $C$, we obtain $\exists b \in \operatorname{supp}(B) \wedge \exists r \in R$, so that $\mu_{r}(b, c)>0$. Similarly, as $b \in \operatorname{supp}(B)$ and $A$ covers $B$, we obtain $\exists a \in \operatorname{supp}(A) \wedge \exists r \in R$, so that $\mu_{r}(a, b)>0$.As $\mu_{r}(a, b)>0 \wedge \mu_{r}(b, c)>0$, we therefore deduce that $a$ exercises an indirect influence on $c$.

Reasoning in the same way about each element of $\operatorname{supp}(C)$ demonstrates the proof.

Note 1. The result we have just proven is also valid for combinations of the three sets $A, B$ and $C$ that are not necessarily fuzzy sets, for example taking $A$ and $C$ as fuzzy sets and $B$ as a non-fuzzy set.

Using the property of coverage, the possible existence of loops is determined in the following result:

Proposition 3. Let $S=(M, R)$ be a complex system, let the fuzzy sets be $A, B \subseteq M$, where $\operatorname{supp}(A)$ is a finite set, so that $\operatorname{supp}(A) \subseteq \operatorname{supp}(B)$. Thus, if $A$ covers $B$, a loop will be included in $\operatorname{supp}(A)$.

Proof. We know that $\operatorname{supp}(A) \subseteq \operatorname{supp}(B)=f_{M}(\operatorname{supp}(A))$, as $A$ covers $B$. Let $n=\operatorname{card}(\operatorname{supp}(A))$, and let $x_{n+1}$ be any finite element of $\operatorname{supp}(A)$. Thus, $x_{n+1} \in f_{M}(\operatorname{supp}(A))$, so that $\exists x_{n} \in \operatorname{supp}(A)$ and $\exists r_{n} \in R$, so that $\mu_{r_{n}}\left(x_{n}, x_{n+1}\right)>0$. In addition, as $x_{n} \in f_{M}(\operatorname{supp}(A)), \exists x_{n-1} \in \operatorname{supp}(A)$ and $\exists r_{n-}$ $1 \in R$, so that $\mu_{r_{n-1}}\left(x_{n-1}, x_{n}\right)>0$.

After $n$ iterations, we obtain a chain of relations $\left\{x_{1}, x_{2}, \ldots \ldots, x_{n}, x_{n+1}\right\}$ and, as the cardinal of $\operatorname{supp}(A)$ is $n$, we obtain $\exists i, j \in\{1,2, \ldots, n+1\}, i<j$, so that $x_{i}=x_{j}$ and therefore the set $\left\{x_{i}, \ldots \ldots, x_{j}\right\}$ forms a loop contained in $\operatorname{supp}(A)$.

Note 2. The above property is also valid if one of the sets is fuzzy and the other is not.

If we combine the two definitions of inclusion described above, we obtain the following result:

Proposition 4. Let there be two fuzzy sets $A, B \subseteq M$, verifying that $A \subseteq B$ (taking into account fuzzy inclusion). Thus, $\operatorname{supp}(A) \subseteq \operatorname{supp}(B)$ (now taking into account ordinary inclusion).

Proof. As $A \subseteq B$, then $\mu_{A}(x) \leq \mu_{B}(x) \forall x \in M$. In addition, if $x \in \operatorname{supp}(A)$, we know that $\mu_{A}(x)>0$ and thus, $\mu_{B}(x) \geq \mu_{A}(x)>0$, so obtaining $x \in \operatorname{supp}(B)$.

\section{Invariability}

This property plays a vital role in studying the evolution of the system in the long term. In previous articles (Esteve and Lloret, 2006a,b) we proved that the abundance of invariable subsets of $M$ can guarantee system behavior with little predisposition to change, and therefore free of upsets. We will now adapt the definition of the invariable subset to our fuzzy environment.

Definition 11. Let $S=(M, R)$ be a complex system and $A \subseteq M$ a fuzzy set. We say that $\operatorname{supp}(A)$ is invariable if $f_{M}(\operatorname{supp}(A)) \subseteq \operatorname{supp}(A)$. 
Note 3. If $A$ is and ordinary set, $A$ is invariable if $f_{M}(A) \subseteq A$.

Example 2. Let $S=(M, R)$ be a complex system where $M$ is a set of cells making up a tissue and let $r \in R$ by the cellular mitosis relation defined by:

$r=\left\{(x, y) \in M x M / \mu_{r}(x, y) \in[0,1]\right\}$ where $\mu_{r}(x, y)>0$ if the cell $y$ descends from cell $x$ by means of mitosis and $\mu_{r}(x, y)=1$ if there were no anomalies in the mitosis process.

We now consider the fuzzy set $A$ associated with the histological tumor grade of each cell $A=\left\langle x, \mu_{A}(x)\right\rangle$, where $\mu_{A}(x)$ is the histological tumor grade of cell $x$. In particular, $\mu_{A}(x)=0$ if cell $x$ is normal.

The support of fuzzy set $A$ is an example of invariability, as the tumor cells are going to appear through mitosis to cells that are also going to have a certain non-null histological tumor grade.

The invariable set condition is not applicable to all its subsets, as can be seen in previously published articles (Esteve and Lloret, 2006a,b). However, there is one very interesting case where this does occur:

Proposition 5. Let $S=(M, R)$ be a complex system and let the fuzzy set $A \subseteq M$ be such that $\operatorname{supp}(A)$ is invariable. Thus, $f_{M}(\operatorname{supp}(A))$ is also invariable.

Proof. Let $a \in f_{M}\left(f_{M}(\operatorname{supp}(A))\right.$. Thus, $\exists c \in f_{M}(\operatorname{supp}(A)) / a \in f_{M}(c)$. As supp(A) is invariable, we obtain $c \in \operatorname{supp}(A)$, and also $a \in f_{M}(\operatorname{supp}(A))$, which proves that $f_{M}(\operatorname{supp}(A))$ is invariable.

Note 4. The above result can also be extended to $f_{M}\left(f_{M}(\operatorname{supp}(A))\right)$, and in this way indefinitely to $f_{M}\left(f_{M}\left(\ldots\left(\ldots\left(f_{M}(\operatorname{supp}(A))\right) \ldots\right) \ldots\right)\right)$.

Proposition 6. Let $S=(M, R)$ be a complex system and let the fuzzy sets be $A, B \subseteq M$, whose supports are both invariable. Thus, $\operatorname{supp}(A) \cup \operatorname{supp}(B)$ for the ordinary union is invariable.

Proof. Let $a \in \operatorname{supp}(A) \cup \operatorname{supp}(B)$, thus $a \in \operatorname{supp}(A)$ or $a \in \operatorname{supp}(B)$. If $a \in \operatorname{supp}(A)$, as $\operatorname{supp}(A)$ is invariable, we obtain $f_{M}(\operatorname{supp}(A)) \subseteq \operatorname{supp}(A)$, and thus $f_{M}(a) \subseteq \operatorname{supp}(A) \cup \operatorname{supp}(B)$. For $a \in \operatorname{supp}(B)$, the same reasoning is applied and we complete the demonstration.

Proposition 7. The fuzzy and ordinary unions and the support satisfy $\operatorname{supp}(A) \cup \operatorname{supp}(B)=$ $\operatorname{supp}(A \cup B)$.

Proof. As $x \in \operatorname{supp}(A) \cup \operatorname{supp}(B) \Leftrightarrow x \in \operatorname{supp}(A)$ or $x \in \operatorname{supp}(B) \Leftrightarrow \mu_{A}(x)>0$ or $\mu_{B}(x)>0 \Leftrightarrow$ $\max \left(\mu_{A}(x), \mu_{B}(x)\right)>0 \Leftrightarrow x \in \operatorname{supp}(A \cup B)$.

Proposition 8 . Let $S=(M, R)$ be a complex system and let the fuzzy sets be $A, B, \subseteq M$, both with invariable supports. Thus, $\operatorname{supp}(A \cup B)$ is invariable.

Proof. Deduced from the two previous propositions.

The same result is obtained with the intersection of two invariable sets and we will now show this. 
Proposition 9. Let $S=(M, R)$ be a complex system and let the fuzzy sets be $A, B \subseteq M$, both with invariable supports. Thus, if $\operatorname{supp}(A) \cap \operatorname{supp}(B) \neq \varnothing$, we see that $\operatorname{supp}(A) \cap \operatorname{supp}(B)$ is invariable for the ordinary intersection.

Proof. Let $x \in \operatorname{supp}(A) \cap \operatorname{supp}(B)$. As $x \in \operatorname{supp}(A)$ y $\operatorname{supp}(A)$ is invariable, $f_{M}(x) \subseteq \operatorname{supp}(A)$. In the same way, with $x \in \operatorname{supp}(B)$ we obtain $f_{M}(x) \subseteq \operatorname{supp}(B)$.

Thus, $f_{M}(x) \subseteq \operatorname{supp}(A) \cap \operatorname{supp}(B)$, giving $f_{M}(\operatorname{supp}(A) \cap \operatorname{supp}(B)) \subseteq \operatorname{supp}(A) \cap \operatorname{supp}(B)$, and thus $\operatorname{supp}(A) \cap \operatorname{supp}(B)$ will be invariable.

Proposition 10. The ordinary and fuzzy intersections and the support satisfy $\operatorname{supp}(A) \cap \operatorname{supp}(B)=\operatorname{supp}(A \cap B)$.

Proof. As $x \in \operatorname{supp}(A) \cap \operatorname{supp}(B) \Leftrightarrow x \in \operatorname{supp}(A)$ and $x \in \operatorname{supp}(B) \Leftrightarrow \mu_{A}(x)>0$ and $\mu_{B}(x)>0 \Leftrightarrow$ $\min \left(\mu_{A}(x), \mu_{B}(x)\right)>0 \Leftrightarrow x \in \operatorname{supp}(A \cap B)$.

Proposition 11. Let $S=(M, R)$ be a complex system and let the fuzzy sets be $A, B \subseteq M$, both with invariable support. Thus, if $\operatorname{supp}(A \cap B) \neq \varnothing$, $\operatorname{supp}(A \cap B)$ is invariable.

Proof. Deduced from the last two propositions.

\section{Relations between coverage and invariability}

We now present a series of results, from different points of view, which prove the relation existing between the concepts of coverage and invariability.

Proposition 12. Let $S=(M, R)$ be a complex system and let the fuzzy sets be $A, B \subseteq M$, both with support invariable. Thus, if $A$ covers $B$, $\operatorname{supp}(A) \cup \operatorname{supp}(B)$ will be invariable.

Proof. Let $x \in \operatorname{supp}(A) \cup \operatorname{supp}(B)$, thus $x \in \operatorname{supp}(A)$ or $x \in \operatorname{supp}(B)$. If $x \in \operatorname{supp}(A)$, as $A$ covers $B$, then $f_{M}(x) \subseteq \operatorname{supp}(B) \subseteq \operatorname{supp}(A) \cup \operatorname{supp}(B)$. In addition, if $x \in \operatorname{supp}(B)$, as this is an invariable set, we get $f_{M}(x) \subseteq \operatorname{supp}(B) \subseteq \operatorname{supp}(A) \cup \operatorname{supp}(B)$. All this proves that $\operatorname{supp}(A) \cup \operatorname{supp}(B)$ is invariable.

Corollary 1. Under the same conditions as the previous Proposition, we have $\operatorname{supp}(A) \cup \operatorname{supp}(B)$ covering $\operatorname{supp}(B)$.

Proof. Trivial, applying properties.

Proposition 13. Let $S=(M, R)$ be a complex system and let the fuzzy sets be $A, B \subseteq M$, so that $A$ covers $B$. Thus, $\operatorname{supp}(A)$ will be invariable $\Leftrightarrow \operatorname{supp}(B) \subseteq \operatorname{supp}(A)$.

Proof. $(\Rightarrow)$ Let $x \in \operatorname{supp}(B)$. As $A$ covers $B$ we obtain $\exists y \in \operatorname{supp}(A)$, so that $x \in f_{M}(y)$. In addition, as $\operatorname{supp}(A)$ is invariable, $x \in f_{M}(y) \subseteq \operatorname{supp}(A)$. In other words, $\operatorname{supp}(B) \subseteq \operatorname{supp}(A)$.

$(\Leftarrow)$ Let $x \in \operatorname{supp}(A)$; as $A$ covers $B$ we obtain $f_{M}(x) \subseteq \operatorname{supp}(B) \subseteq \operatorname{supp}(A)$. Thus, $f_{M}(\operatorname{supp}(A) \subseteq$ $\operatorname{supp}(A)$ and $\operatorname{supp}(A)$ will be invariable.

One obvious result of the previous Proposition is shown below. 
Corollary 2. Let $S=(M, R)$ be a complex system and let the fuzzy set be $A \subseteq M$, so that $A$ covers itself. Thus, $\operatorname{supp}(A)$ is invariable.

Proof. Trivial, replacing $A$ with $B$ in the previous Proposition.

We will now examine a known result under different premises.

Proposition 14. Let $S=(M, R)$ be a complex system and let the fuzzy sets be $A, B \subseteq M$, so that $A$ covers $B$ and $B$ covers $A$. Thus, $\operatorname{supp}(A) \cup \operatorname{supp}(B)$ is invariable.

Proof. Let $x \in \operatorname{supp}(A) \cup \operatorname{supp}(B)$, thus $x \in \operatorname{supp}(A)$ or $x \in \operatorname{supp}(B)$. If $x \in \operatorname{supp}(A)$, we obtain $f_{M}(x) \subseteq \operatorname{supp}(B) \subseteq \operatorname{supp}(A) \cup \operatorname{supp}(B)$. In addition, if $x \in \operatorname{supp}(B)$, we obtain $f_{M}(x) \subseteq \operatorname{supp}(A) \subseteq \operatorname{supp}(A) \cup \operatorname{supp}(B)$.

Thus, $\quad \forall x \in \operatorname{supp}(A) \cup \operatorname{supp}(B) \quad$ verifies $\quad$ that $\quad f_{M}(x) \subseteq \operatorname{supp}(A) \cup \operatorname{supp}(B)$, giving $f_{M}(\operatorname{supp}(A) \cup \operatorname{supp}(B)) \subseteq \operatorname{supp}(A) \cup \operatorname{supp}(B)$, and therefore $\operatorname{supp}(A) \cup \operatorname{supp}(B)$ will be invariable.

We obtain a similar but more enlarged result with the following corollary.

Corollary 3. Let $S=(M, R)$ be a complex system and let the fuzzy sets be $A_{0}, A_{1}, \ldots ., A_{n} \subseteq M$, so that $A_{0}=A_{n} \quad$ and $A_{i}$ cover $A_{i+1} \forall i=0,1, \cdots, n-1$. Thus, $\operatorname{supp}\left(A_{0}\right) \cup \operatorname{supp}\left(A_{1}\right) \cup \ldots \ldots \cup \operatorname{supp}\left(A_{n-1}\right)$ is invariable.

Proof. Trivial, applying the known properties and using the same reasoning as in the previous Proposition.

We finish with an important result that shows the fundamental relation between the loops and any invariable set.

Proposition 15. Let $S=(M, R)$ a fuzzy complex system and let $A, L \subseteq M$ be fuzzy sets, so that $\operatorname{supp}(A)$ is invariable and $\operatorname{supp}(L)$ is a loop. Thus, it can only be that $\operatorname{supp}(L) \subseteq \operatorname{supp}(A)$ or that $\operatorname{supp}(A)$ and $\operatorname{supp}(L)$ are disjointed.

Proof. Let $x \in \operatorname{supp}(L)$. We can find that $x \in \operatorname{supp}(A)$ or $x \notin \operatorname{supp}(A)$. Firstly, suppose that $x \in \operatorname{supp}(A)$. As $\operatorname{supp}(A)$ is invariable, we will have $f_{M}(x) \subseteq \operatorname{supp}(A)$. In other words, the element following $x$ in the loop will also belong to $\operatorname{supp}(A)$. Using the same reasoning, the following element will also belong to $\operatorname{supp}(A)$ and successively until we reach our element $x$.All elements in the loop therefore belong to $\operatorname{supp}(A)$ and we obtain that $\operatorname{supp}(L) \subseteq \operatorname{supp}(A)$. Now suppose that $x \notin \operatorname{supp}(A)$. In this case, if the element following $x$ in the loop is an element of $\operatorname{supp}(A)$, then the successive elements in the loop (note that as it is a loop, we are talking about all its elements) will also belong to $\operatorname{supp}(A)$ and, as $\operatorname{supp}(A)$ is invariable, our element $x$ will also do so, thus contradicting our initial supposition.

So, if $x \in \operatorname{Supp}(L)$, we have $x \notin \operatorname{supp}(A)$, in other words, both sets will be disjointed.

\section{Conclusion}

The concepts of coverage and invariability from a discrete and fuzzy point of view are adaptations of the definitions made by the authors (Alseda et. al. 2000; Block and Coppel, 1992) for the case of continuous systems. 
These concepts are only obtained with an iteration of the associated relation structures, thus focusing on the direct or immediate influences between the different elements making up the complex system.

These elements are defined separately and analyzing all possibilities according to whether the sets in question are fuzzy or not, underlining their implications with the loop concept and seeing what results are obtained. The concept of coverage has revealed properties between sets like neighboring relations between them. As regards invariability, the results have shown little or no predisposition to disorder in the complex systems containing invariable fuzzy sets.

\section{References}

Alseda, LL., Llibre M., Misiurewicz, M. (2000). Combinatorial dynamics and entropy in dimension one. World Scientific Publishing Co., Inc., River Edge, N.J.

Atanassov, K. T. (1986). 'Intuitionistic fuzzy sets,’ Fuzzy Sets and Systems, 20 (1), 87-96.

Block, L., and Coppel, W.A. (1992). Dynamics in one dimension. Spinger-Verlag, Berlin.

Blue, M., Blush, B., Puckett, J. (2002). 'Unified approach to fuzzy graph problems, ' Fuzzy Sets and Systems, 125 (3), 355-368.

Chen, S. M., and Tan, J.M. (1994).'Handling Multicriteria Fuzzy Decision-Making Problems Based on Vague Theory,`Fuzzy Sets and Systems, 67, 163-172.

Ding, J. and Chou, C. (2013).' An evaluation Model of Quantitative and Qualitative Fuzzy Multi-Criteria Decision-Making Approach for Location Selection of Transshipment Ports, Mathematical Problems in Engineering, vol. 2013, ID. 783105, 1-12.

Esteve, P.F., and Lloret, M. (2006a). 'Coverage and invariability by structural functions,' International Journal of General systems, 35, 699-706.

Esteve, P.F., and Lloret, M. (2006b). 'Coverage, Invariability and orbits by structural functions Kybernetes, 35 (7-8), 1236-1240.

Gunduz, C., and Bayramov, S. (2013). 'Some Results on Fuzzy Soft Topological Spaces,' Mathematical Problems in Engineering. 2013, ID. 835308, 1-10.

Hong, D.H., and Choi, C.H. (2000), 'Multicriteria Fuzzy Decision-Making Problems Based on Vague Set Theory,’ Fuzzy Sets and Systems, 114, 103-113.

Kikuchi, S., and Kronprasert, N. (2012). Effects of data quality and quantity in systems modelling: a case study. International Journal of General Systems, 41 (7), 679-711.

Kóczy, L. T. (1992). 'Fuzzy graphs in the evaluation and optimization of networks, ' Fuzzy Sets and Systems, 46 (3), 307-319.

Liu, H.W., and Wang, G.J. (2007), 'Multi-criteria Decision-Making Methods Based on Intuitionistic Fuzzy Sets,’ European Journal of Operational Research, 179, 220-233.

Molodtsov, D. (1999). 'Soft set theory-first results,' Computers and Mathematics with Applications, 37 (4-5), 19-31. 
Pawlak, Z. (1982). 'Rough sets,' International Journal of Computer \& Information Sciences, 11 (5), 341-356.

Raghuvanshi, P. S. and Kumar, S. (1999).' On the Structurating of Systems with Fuzzy Relations, IEEE Transactions on Systems, Man, and Cybernetics-Part B: Cybernetics, 29 (4), 547-553.

Tazaki, E. and Amagasa, M. (1979). 'Structural modeling in a class of systems using fuzzy sets theory,' Fuzzy Sets and Systems, 2 (1), 87-103.

Yamashita, T. (1997). 'On a support system for human decision making by the combination of fuzzy reasoning and fuzzy structural modeling,' Fuzzy Sets and Systems, 87 (3), 257-263.

Ye, J. (2010), 'Fuzzy Decision-Making Method Based on the Weighted Correlation Coefficient Under Intuitionistic Fuzzy Environment,' European Journal of Operational Research, 205, 202-204.

Ye, J. (2012), 'Multicriteria group decision-making method using the distances-based similarity measures between intuitionistic trapezoidal fuzzy numbers,' International Journal of General Systems, 41 (7), 729-739.

Zadeh, L. A. (1965). 'Fuzzy sets,`Information and Control, 8 (3), 338-353.

Zadeh, L. A. (1999). 'Fuzzy logic and the calculi of fuzzy rules, fuzzy graphs, and fuzzy probabilities, 'Computers \& Mathematics with Applications. 37 (11-12), 35.

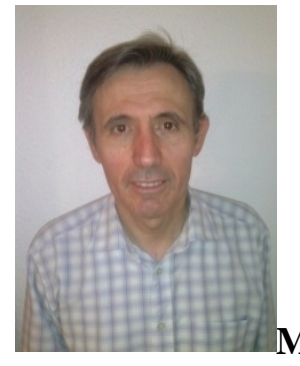

Miguel Lloret-Climent has a doctorate in Mathematics from the University of Alicante (Spain) and this currently professor of Applied Mathematics in the University of Alicante. He is author and co-author of several papers in journals and proceedings. He is devoted to general research on the Theory of General Systems and Biocybernetics.

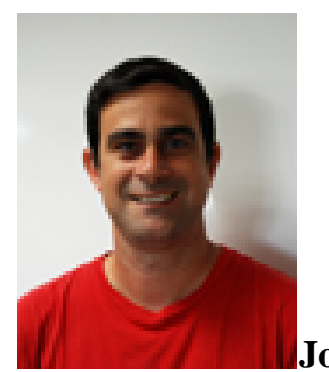

Josué-Antonio Nescolarde-Selva graduated in Mathematics from the University of Havana (Cuba) in 1999. He won the award: Gold Title at the University of Havana, Cuba in 1999. He received the PhD degree in Mathematics from the University of Alicante (Spain) in 2010. Since 2002, he has been working in the Department of Applied Mathematics, University of Alicante, Spain. He is author 
and co-author of several papers in journals and books. He is devoted to research on the Theory of Systems and Belief Systems.

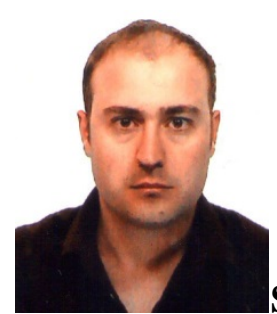

Sergio Pérez-Gonzaga graduated in Mathematics from the University of Alicante (Spain). He works as a secondary mathematics teacher and he is currently working on his $\mathrm{PhD}$ at the Department of Applied Mathematics, University of Alicante, Spain. 